\title{
Role of antioxidant enzymes and plant performances towards heavy metal stress in ornamental sunflower by vermicompost implementation
}

\author{
F. Mojdehi' ${ }^{1}$, M. Taghizadeh ${ }^{2}$, A.H. Baghaie ${ }^{3}$, M. Changizi $^{4}$ and S. Khaghani ${ }^{4}$ \\ ${ }^{1}$ Department of Horticultural Science, Arak Branch, Islamic Azad University, Arak, Iran \\ 2 Department of Horticultural Science, Faculty of Agriculture and Environment Science, Arak University, Arak, Iran \\ ${ }^{3}$ Department of Soil Science, Arak Branch, Islamic Azad University, Arak, Iran \\ ${ }^{4}$ Department of Agronomy and Plant Breeding, Arak Branch, Islamic Azad University, Arak, Iran
}

\section{Summary}

Almost hyperaccumulators have a short growth period and some are edible crops with low-biomass. So, in this study, the potential of ornamental sunflower to remedy contaminated soil was evaluated. The experiment was set in as factorial arrangements in a completely randomized design where the treatments were 3 concentrations vermicompost $(0,15$, $\left.30 \mathrm{~kg} \mathrm{~m}^{-2}\right), 3 \mathrm{~Pb}$ concentrations $(0,400,600 \mathrm{ppm})$ and 4 Cd concentrations $(0,5,10,15 \mathrm{ppm})$. The oxidative enzyme activities were greatly influenced by the presence of vermicompost and various contaminant levels. Treatment of $\mathrm{Pb}$ and $\mathrm{Cd}$ without vermicompost application, increased superoxide dismutase and catalase activity 27- and 12-folds, respectively, in comparison with control, but in the presence of both metal treatments accompany with $30 \mathrm{~kg} \mathrm{~m}^{-2}$ vermicompost, enzyme activity was increased 15 times in superoxide dismutase and 9 times in catalase more than the control. The addition of vermicompost amendments has been shown to increase soil microbial activity. The results show that the translocation factor was not greater than 1 in ornamental sunflower at none of the studied treatments, but the biological concentration factor was more than 1. Our results indicated that ornamental sunflower showed tolerance to $\mathrm{Pb}$ and $\mathrm{Cd}$ contamination and Helianthus annuus 'Sungold' was more favorable for $\mathrm{Pb}$ uptake compared to $\mathrm{Cd}$.

Keywords

ornamental plant, lead, cadmium, SOD, CAT, organic amendment

\section{Introduction}

Nowadays, soil contamination by heavy metals has posed a serious challenge in the industrial area soils worldwide. Heavy metal contamination of the soil is different from the air or water contaminations as heavy metals won't easily leach away upon their accumulation in the soil, giving rise to their higher durability in soils rather than any other parts of the biosphere (Lasat, 2002). Human activities have resulted in extensive soil contamination by lead $(\mathrm{Pb})$ and cadmium (Cd). High concentrations of heavy metals will decrease the nutrients uptake, prevent enzymatic activity and induce oxidative stress (changes in the enzymes involved in the antioxidant defense system) (Majer et al., 2002; Agrawal

\section{Significance of this study}

What is already known on this subject?

- Nowadays, soil contamination by heavy metals has posed a serious challenge in the industrial area soils worldwide. Phytoremediation is a promising approach for soil improvement which removes heavy metals. Most of the research works in the field of phytoremediation have been so far focused on vegetables and forage crops which may bring about the risk of heavy metals entrance in the food chain.

What are the new findings?

- Ornamental sunflower (Helianthus annuus 'Sungold') as a non-edible plant was chosen based on their high biomass and fast growth rate. The presence of organic matter in the soil has beneficial influences and improves its phytoremediation properties. The oxidative enzyme activities were greatly influenced by the presence of vermicompost and various $\mathrm{Pb}$ and Cd concentrations. Ornamental sunflower showed tolerance to $\mathrm{Pb}$ and $\mathrm{Cd}$ contamination and it was more favorable for $\mathrm{Pb}$ uptake compared to $\mathrm{Cd}$.

What is the expected impact on horticulture?

- Helianthus annuus 'Sungold' showed tolerance toward $\mathrm{Pb}$ and $\mathrm{Cd}$ contamination and this ornamental plant was more favorable for $\mathrm{Pb}$ and $\mathrm{Cd}$ uptake. Therefore, it can be recommended for the remediation of soil cocontaminated in landscape of polluted area.

and Sharma, 2006). The antioxidative system uses the antioxidant enzymes (mainly including superoxide dismutase (SOD), catalase (CAT), peroxidase (POD), and glutathione reductase (GR) to scavenge the reactive oxygen species (ROS) and thereby, prevent oxidative damage (Sandalio et al., 2009; Aibibu et al., 2010). Heavy metals can greatly threaten the plant and human health cycles upon their entrance into the soil. In this regard, a huge deal of attention has been paid to control and manage the heavy metals content of the soils to prevent their entrance into the food chain and groundwater (Farouk et al., 2011). Phytoremediation is a promising approach for soil improvement which removes heavy metals through absorption (Ghori et al., 2016). As one of the phytoremediation technologies, phytoextraction involves the transportation of the heavy metal from the polluted water 
source or terrestrial source to the phyto-biomass. This is a biological process for the decontamination of heavy metal (Singer et al., 2007). Each type of contaminant requires a different phytoremediation process which may include a wide variety of plants. Various plant species can hyper-accumulate the heavy metals in their tissues. 45 families of plants are able to accumulate heavy metals (Ullah et al., 2015). Most of these plants suffer from several deficiencies, for instance, hyperaccumulator plants have short growth duration and are low-biomass producers. On the other hand, high-biomass plants are generally sensitive to high concentrations of heavy metals and have less ability to transport metals from roots to shoots (Muszynska and Hanus-Fajerska, 2015). Thus, researches aimed at identifying the native hyperaccumulators, and non-edible plants (such as ornamental plants) with high biomass production rate during the growth period and elevated metals uptake and root-shoot transport have been increased.

Most of the research works in the field of phytoremediation have been so far focused on vegetables and forage crops which may bring about the risk of heavy metals entrance in the food chain. In this regard, studies on tolerance, absorption, and accumulation of heavy metals by different plant species sound crucial. Ornamental plant sunflower (Helianthus annuus 'Sungold') belongs to the family of Asteraceae. It is grown in a wide range of soil types as a bedding plant outside, pot flower and cut-flower.

Previous studies have revealed the high tolerance of sunflower (as an edible crop) against heavy metals (Pilon-Smits, 2005; Chirakkara and Reddy, 2015; Rizwan et al., 2016; Govarthanana et al., 2018). So, in this study, in addition to the ornamental characteristics of sunflower, its potential ability to improve the contaminated soil will be investigated. For this purpose, the role of vermicompost in $\mathrm{Pb}$ and $\mathrm{Cd}$ absorption by ornamental sunflower and the effects of heavy metals contamination on antioxidant characteristics and phytoremediation indices were addressed.

\section{Materials and methods}

\section{Soil and vermicompost sampling, characterization, and processing}

The physicochemical parameters of soil samples were analyzed by standard methods and their organic carbon (OC), pH, EC, lime, cation exchange capacity (CEC), total nitrogen and total phosphorus, transferable potassium, total nitrogen, soil texture, and extractable heavy metals $(\mathrm{Cd}$ and $\mathrm{Pb}$ ) were determined. The results of physical and chemical analyses of the studied soils are presented in Table 1 . The concentrations of $\mathrm{Pb}$ and $\mathrm{Cd}$ were below the detection limit of the applied instrument. The cow vermicompost with min- imum heavy metal content was used as the organic fertilizer whose analysis results are listed in Table 1 . The soil sample was mixed with $\mathrm{CdCl}_{2}$ at $0,5,10,15 \mathrm{ppm}$ and $\mathrm{Pb}\left(\mathrm{NO}_{3}\right)_{2}$ at 0 , $400,600 \mathrm{ppm}$. Then it was equilibrated in a greenhouse for one month at $60 \%$ water holding capacity (WHC) prior to the experiment. The polluted soil was filtered through a 2-mm stainless steel sieve. The vermicompost was then added in different treatments $\left(0,15,30 \mathrm{~kg} \mathrm{~m}^{-2}\right)$. The plastic pots were filled with $5 \mathrm{~kg}$ soil and different proportions of vermicompost to soil were added: concentrations of 0,50 , and $100 \mathrm{~g}$ per pot.

\section{Greenhouse experiments}

Seeds of ornamental sunflower (Helianthus annuus 'Sungold') were obtained from Hem Zaden BV. Greenhouse pot culture experiments were conducted to study the effect of vermicompost on $\mathrm{Cd}$ and $\mathrm{Pb}$ phytoremediation potential (uptake) of the ornamental sunflower. The heavy metal-contaminated soil-vermicompost was employed as the potting media. The seeds were sown in soil-vermicompost media and irrigated with distilled water. To prevent the loss of nutrients and trace elements from the pots, plastic trays were placed under each pot and the leached fluid was returned to the corresponding pots. After two weeks, the seedlings were thinned to one plant per pot and grown. Distilled water was added as needed.

\section{Harvest and determination of heavy metals in plant and soil}

After 60 days of cultivation, the plants were gently removed from the pots. They were then cut into roots and shoots, washed with tap water, and then with deionized water to remove any residual soil or dust and dried at room temperature. Shoot and roots samples were dried in an oven at $60^{\circ} \mathrm{C}$ for $48 \mathrm{~h}$ and their dry matter was measured. The plant materials were ground by an electric grinder into fine powders. They were then kept for further measurement of heavy metals. $0.5 \mathrm{~g}$ of the sieved plant samples were digested with a mixture of $\mathrm{HCl} / \mathrm{HNO}_{3}(3: 1, \mathrm{v} / \mathrm{v}) . \mathrm{Pb}$ and $\mathrm{Cd}$ contents (ppm) of the digested dry plants were determined using atomic absorption spectrophotometry (AAS, Perkin Elmer) as described by Lindsay and Norvell (1978). The soil was digested in order to determine the $\mathrm{Pb}$ and $\mathrm{Cd}$ content by an atomic absorption spectrophotometer (Sabiene et al., 2004) as well. In this study, phytoremediation factors of $\mathrm{Pb}$ and $\mathrm{Cd}$ were also assessed. The ability of ornamental sunflower for heavy metal accumulation and upwards translocation was determined by calculating the tolerance index (TI), biological concentration factor (BCF), biological accumulation coefficient (BAC), and translocation factor (TF) (Martinez-Sanchez et al., 2012; Giri and Patel, 2012).

TABLE 1. Some physiochemical properties of experimental soil and vermicompost fertilizer.

\begin{tabular}{lclc}
\hline Soil & & Vermicompost & \\
\hline $\mathrm{pH}$ & 7.3 & $\mathrm{pH}$ & 6.9 \\
$\mathrm{EC}\left(\mathrm{dS}^{-1}\right)$ & 1.1 & $\mathrm{EC}\left(\mathrm{dS}^{-1}\right)$ & 21.4 \\
Organic carbon $(\%)$ & 0.1 & Organic carbon $(\%)$ & 1.5 \\
Soil texture & Loamy & Total N $\%)$ & 125.17 \\
Lime $(\%)$ & 8 & Available Zn $\left(\mathrm{mg} \mathrm{kg}^{-1}\right)$ & 1.23 \\
$\mathrm{~Pb}\left(\mathrm{mg} \mathrm{kg}^{-1}\right)$ & $\mathrm{ND}$ & Available P $\left(\mathrm{mg} \mathrm{kg}^{-1}\right)$ & 3.4 \\
$\mathrm{Cd}\left(\mathrm{mg} \mathrm{kg}^{-1}\right)$ & $\mathrm{ND}$ & $\mathrm{Pb}\left(\mathrm{mg} \mathrm{kg}^{-1}\right)$ & 1.1 \\
$\mathrm{CEC}\left(\mathrm{c} \mathrm{mol} 100 \mathrm{~g} \mathrm{~g}^{-1}\right.$ soil) & 13.3 & $\mathrm{Cd}\left(\mathrm{mg} \mathrm{kg}^{-1}\right)$ & \\
\hline
\end{tabular}




\section{Measurement of antioxidant enzymes and microbial respiration}

The total SOD activity was evaluated by measuring its ability to inhibit the photochemical reduction of nitrotetrazolium blue chloride (NBT), as described by Rios-Gonzales et al. (2002). The CAT activity was also measured by $\mathrm{H}_{2} \mathrm{O}_{2}$-consumption as previously described by Aebi (1984). SOD and CAT activities were determined by monitoring the decline in the absorbance at $420 \mathrm{~nm}$ and $240 \mathrm{~nm}$ by spectrophotometer, respectively. The basal soil microbial respiration was determined as evolved $\mathrm{CO}_{2}$ using the method developed by Alef and Nannipieri (1995).

\section{Design and statistical analysis}

The experiment was set in as factorial arrangements in a completely randomized design (CRD) $(3 \times 3 \times 4)$ where the treatments were the 3 concentrations vermicompost, $3 \mathrm{~Pb}$ concentrations and $4 \mathrm{Cd}$ concentrations. Each treatment consisted of three replicates for statistical purposes. All the results were statistically analyzed using the SAS program (v. 9.1). Means of three replicates were subjected to one-way ANOVA. Duncan's Multiple Range Test (DMRT) was also used to compare the mean values and determine the significance of statistical differences in treatments at $p \leq 0.05$. The obtained data were reported as mean plus the standard deviation (X \pm S.D.).

TABLE 2. Effect of vermicompost amendment on biomass, antioxidant activity and microbial respiration in ornamental sunflower under lead $(\mathrm{Pb})$ and cadmium $(\mathrm{Cd})$ stress. Values are mean \pm SD of three replicates. In each column, numbers followed by the same letter are not significantly different according to Duncan's test $(\mathrm{P}<0.05)$.

\begin{tabular}{|c|c|c|c|c|c|c|c|}
\hline $\begin{array}{l}\text { Vermi- } \\
\text { compost } \\
\left(\mathrm{kg} \mathrm{m}^{-2}\right)\end{array}$ & $\begin{array}{c}\mathrm{Pb} \\
(\mathrm{ppm})\end{array}$ & $\begin{array}{c}\mathrm{Cd} \\
(\mathrm{ppm})\end{array}$ & $\begin{array}{l}\text { FW } \\
(g)^{\dagger}\end{array}$ & $\begin{array}{l}\text { DW } \\
(\mathrm{g})\end{array}$ & $\begin{array}{c}\mathrm{SOD} \\
\text { (U mg-1 protein) }\end{array}$ & $\begin{array}{c}\text { CAT } \\
\text { (U mg-1 protein) }\end{array}$ & $\begin{array}{c}\mathrm{CO} \\
\text { (mg C-CO } \mathrm{kg}^{-1} \\
\text { soil) }\end{array}$ \\
\hline 0 & 0 & 0 & $0.72 \pm 0.45 f g h i$ & $0.49 \pm 0.29$ efghi & $0.08 \pm 0.005 v w$ & $0.1 \pm 0.005 u v$ & $1.11 \pm 0.01 \mathrm{z}^{\dagger \dagger}$ \\
\hline 0 & 0 & 5 & $0.66 \pm 0.36 f g h i$ & $0.43 \pm 0.22$ efghi & $0.31 \pm 0.02 r$ & $0.41 \pm 0.02 q$ & $4.24 \pm 0.02 \mathrm{~s}$ \\
\hline 0 & 0 & 10 & $0.32 \pm 0.11 \mathrm{ghi}$ & $0.22 \pm 0.10$ hig & $1.21 \pm 0.01 \mathrm{~h}$ & $1.11 \pm 0.01 \mathrm{k}$ & $5.0 \pm 0.05 q$ \\
\hline 0 & 0 & 15 & $0.83 \pm 0.70 f g h i$ & $0.51 \pm 0.43$ efghi & $1.38 \pm 0.02 f$ & $1.49 \pm 0.01 \mathrm{~g}$ & $6.11 \pm 0.02$ \\
\hline 0 & 400 & 0 & $0.15 \pm 0.13 i$ & $0.09 \pm 0.07 i$ & $0.13 \pm 0.01 u$ & $0.32 \pm 0.01 \mathrm{rst}$ & $1.5 \pm 0.03 x$ \\
\hline 0 & 400 & 5 & $0.08 \pm 0.007 i$ & $0.05 \pm 0.002 i$ & $0.52 \pm 0.010$ & $0.7 \pm 0.030$ & $5.1 \pm 0.01 p$ \\
\hline 0 & 400 & 10 & $0.12 \pm 0.02 i$ & $0.08 \pm 0.01 i$ & $1.62 \pm 0.02 d$ & $2.05 \pm 0.01 d$ & $6.3 \pm 0.04 \mathrm{k}$ \\
\hline 0 & 400 & 15 & $0.49 \pm 0.17 \mathrm{ghi}$ & $0.30 \pm 0.01 \mathrm{fghi}$ & $1.91 \pm 0.01 b$ & $2.21 \pm 0.01 \mathrm{c}$ & $7.5 \pm 0.03 \mathrm{fg}$ \\
\hline 0 & 600 & 0 & $0.64 \pm 0.64$ fghi & $0.38 \pm 0.40$ fghi & $0.28 \pm 0.005$ & $0.44 \pm .03 q$ & $2.1 \pm 0.02 v$ \\
\hline 0 & 600 & 5 & $0.32 \pm 0.13 \mathrm{ghi}$ & $0.19 \pm 0.07 \mathrm{hi}$ & $0.72 \pm 0.02 n$ & $0.98 \pm 0.03 \mid$ & $5.8 \pm 0.02 m$ \\
\hline 0 & 600 & 10 & $0.39 \pm 0.27 \mathrm{ghi}$ & $0.22 \pm 0.15 \mathrm{hi}$ & $1.81 \pm 0.01 \mathrm{c}$ & $2.28 \pm 0.02 b$ & $7.1 \pm 0.03 \mathrm{~h}$ \\
\hline 0 & 600 & 15 & $0.29 \pm 0.15 \mathrm{ghi}$ & $0.16 \pm 0.08 \mathrm{hi}$ & $2.18 \pm 0.0 \mathrm{a}$ & $2.41 \pm 0.03 a$ & $8.29 \pm 0.02 e$ \\
\hline 15 & 0 & 0 & $0.51 \pm 0.25 \mathrm{ghi}$ & $0.28 \pm 0.13$ fghi & $0.04 \pm 0.00 x y$ & $0.17 \pm 0.01 v$ & $1.3 \pm 0.02 y$ \\
\hline 15 & 0 & 5 & $5.46 \pm 0.81 a$ & $3.08 \pm 0.44 a$ & $0.21 \pm 0.01 t$ & $0.31 \pm 0.01 \mathrm{st}$ & $4.93 \pm 0.02 r$ \\
\hline 15 & 0 & 10 & $5.21 \pm 1.65 a b$ & $3.10 \pm 0.89 a$ & $0.72 \pm 0.02 n$ & $0.91 \pm 0.01 \mathrm{~m}$ & $5.34 \pm 0.030$ \\
\hline 15 & 0 & 15 & $3.22 \pm 2.41 \mathrm{bcde}$ & $1.80 \pm 1.35 \mathrm{bcde}$ & $1.04 \pm 0.03 k$ & $1.11 \pm 0.01 \mathrm{k}$ & $6.40 \pm 0.02 k$ \\
\hline 15 & 400 & 0 & $1.5 \pm 0.82$ efghi & $0.86 \pm 0.42$ defghi & $0.08 \pm 0.00 v w$ & $0.22 \pm 0.02 u$ & $1.91 \pm 0.01 w$ \\
\hline 15 & 400 & 5 & $1.2 \pm 0.9$ efghi & $0.71 \pm 0.50$ defghi & $0.32 \pm 0.01 r$ & $0.51 \pm 0.01 p$ & $5.49 \pm 0.01 n$ \\
\hline 15 & 400 & 10 & $5.1 \pm 4.0 \mathrm{ab}$ & $3.09 \pm 2.47 a$ & $0.96 \pm 0.01 \mid$ & $1.30 \pm 0.01 \mathrm{i}$ & $6.71 \pm 0.02 j$ \\
\hline 15 & 400 & 15 & $1.4 \pm 0.41$ efghi & $0.87 \pm 0.47$ defghi & $1.32 \pm 0.02 \mathrm{~g}$ & $1.83 \pm 0.03 e$ & $8.45 \pm 0.04 d$ \\
\hline 15 & 600 & 0 & $4.02 \pm 1.62 \mathrm{abcd}$ & $2.33 \pm 0.95 \mathrm{abc}$ & $0.10 \pm 0.005$ & $0.33 \pm 0.05$ rs & $2.42 \pm 0.03 \mathrm{v}$ \\
\hline 15 & 600 & 5 & $5.42 \pm 1.32 a$ & $3.20 \pm 0.82 a$ & $0.49 \pm 0.01$ & $0.72 \pm 0.20$ & $6.13 \pm 0.011$ \\
\hline 15 & 600 & 10 & $1.95 \pm 0.4$ defghi & $1.14 \pm 0.28$ cdefghi & $1.15 \pm 0.04$ & $1.49 \pm 0.06 \mathrm{~g}$ & $7.45 \pm 0.04 \mathrm{~g}$ \\
\hline 15 & 600 & 15 & $0.26 \pm 0.13 \mathrm{hi}$ & $0.15 \pm 0.07$ & $1.52 \pm 0.02 u v$ & $2.04 \pm 0.01 d$ & $10.20 \pm 0.09 b$ \\
\hline 30 & 0 & 0 & $2.19 \pm 1.1$ defghi & $1.26 \pm 0.68$ cdefghi & $0.01 \pm 0.005 y$ & $0.08 \pm 0.01 w$ & $1.60 \pm 0.01 x$ \\
\hline 30 & 0 & 5 & $4.94 \pm 0.27 a b c$ & $2.88 \pm 0.22 a b$ & $0.20 \pm 0.005 t$ & $0.22 \pm 0.01 u$ & $5.22 \pm 0.01 \mathrm{po}$ \\
\hline 30 & 0 & 10 & $1.39 \pm 1.10$ efghi & $0.77 \pm 0.61$ defghi & $0.52 \pm 0.020$ & $0.75 \pm 0.020$ & $5.71 \pm 0.01 \mathrm{~m}$ \\
\hline 30 & 0 & 15 & $2.22 \pm 1.6$ defghi & $1.25 \pm 0.95$ & $0.07 \pm 0.01 \mathrm{vw}$ & $0.81 \pm 0.03 n$ & $7.59 \pm 0.01 f$ \\
\hline 30 & 400 & 0 & $3.57 \pm 1.90$ abcde & $2.04 \pm 1.11 \mathrm{abcd}$ & $0.05 \pm .005 w x$ & $0.2 \pm 0.005 u v$ & $2.13 \pm 0.01 \mathrm{v}$ \\
\hline 30 & 400 & 5 & $2.92 \pm 2.15 \mathrm{cdef}$ & $1.61 \pm 1.17 \mathrm{bcdefg}$ & $0.34 \pm 0.02 r$ & $0.35 \pm 0.005 r$ & $5.70 \pm 0.01 \mathrm{~m}$ \\
\hline 30 & 400 & 10 & $2.65 \pm 1.10 \mathrm{defg}$ & $1.51 \pm 0.63 \mathrm{cdefgh}$ & $0.81 \pm 0.01 \mathrm{~m}$ & $1.13 \pm 0.03$ & $6.94 \pm 0.02 i$ \\
\hline 30 & 400 & 15 & 2.600 .51 defgh & $1.44 \pm 0.26$ cdefghi & $1.11 \pm .01 \mathrm{j}$ & $1.35 \pm 0.04$ & $9.55 \pm 0.05 c$ \\
\hline 30 & 600 & 0 & $2.07 \pm 1.0$ defghi & $1.23 \pm 0.61$ cdefghi & $0.08 \pm 0.005 v w$ & $0.28 \pm 0.00 t 5$ & $2.71 \pm 0.02 t$ \\
\hline 30 & 600 & 5 & $1.44 \pm 0.54 \mathrm{efghi}$ & $0.84 \pm 0.32$ defghi & $0.39 \pm 0.01 q$ & $0.49 \pm 0.01 p$ & $6.30 \pm 0.02$ \\
\hline 30 & 600 & 10 & $0.84 \pm 0.30$ fghi & $0.48 \pm 0.16$ efghi & $0.97 \pm 0.02$ & $1.25 \pm 0.01 j$ & $7.60 \pm 0.04 f$ \\
\hline 30 & 600 & 15 & $2.97 \pm 0.36 \mathrm{cdef}$ & $1.67 \pm 0.28 \mathrm{bcdef}$ & $1.20 \pm 0.03 \mathrm{~h}$ & $1.73 \pm 0.02 f$ & $12.13 \pm 0.1 \mathrm{a}$ \\
\hline
\end{tabular}

† FW: Fresh weight; DW: Dry weight; SOD: superoxide dismutase enzyme; CAT: catalase enzyme; CO: microbial respiration.

tt Values followed in each column by the same letter were not significantly different at $5 \%$ level. 


\section{Results}

The amount of biomass produced during phytoremediation is the most prominent characteristic in this technique. The results of this experiment showed that the application of different levels of vermicompost in the soil polluted by cadmium and lead enhanced the fresh and dry weight of ornamental sunflower during the cultivation period. The application of vermicompost at the concentration of $15 \mathrm{~kg} \mathrm{~m}^{-2}$ led to the highest of fresh ( $5 \mathrm{~g}$ ) and dry ( $3 \mathrm{~g}$ ) weights of ornamental sunflower during the cultivation period in the soil contaminated with $0 \mathrm{ppm}$ and $600 \mathrm{ppm}$ lead and $5 \mathrm{ppm}$ cadmium. Conversely, an increase of $\mathrm{Pb}$ and $\mathrm{Cd}$ concentrations in the soil resulted in a reduction in biomass production. The least biomass production (0.05-0.15 g) was measured in the treatment containing $400 \mathrm{ppm}$ lead and $0-10 \mathrm{ppm}$ cadmium and the one with no vermicompost amendment. The effect of cadmium concentrations on the decrease of the dry weight was more noticeable than that of lead (Table 2).

Moreover, oxidative enzyme activities were greatly influenced by the presence of vermicompost and various contaminant levels. CAT and SOD activities were decreased as a result of vermicompost enhancement, but the presence of cadmium and lead in the polluted soil caused abiotic stress and induced the CAT and SOD activity. The maximum activity of both enzymes was measured in the treatment containing the highest concentration of metal contaminants, i.e., $600 \mathrm{ppm}$ lead and $15 \mathrm{ppm}$ cadmium and without vermicompost amendment (2.18 for SOD and 2.41 for CAT $\mathrm{U} \mathrm{mg}^{-1}$ protein).

TABLE 3. Effect of vermicompost amendment on BAC, BCF and TF parameters for lead in ornamental sunflower under lead $(\mathrm{Pb})$ and cadmium $(\mathrm{Cd})$ stresses. Values are mean \pm SD of three replicates. In each column, numbers followed by the same letter are not significantly different according to Duncan's test $(\mathrm{P}<0.05)$.

\begin{tabular}{|c|c|c|c|c|c|}
\hline $\begin{array}{l}\text { Vermicompost } \\
\left(\mathrm{kg} \mathrm{m}^{-2}\right)\end{array}$ & $\begin{array}{c}\mathrm{Pb} \\
(\mathrm{ppm})\end{array}$ & $\begin{array}{c}\mathrm{Cd} \\
(\mathrm{ppm}) \\
\end{array}$ & $\mathrm{BAC} \mathrm{Pb}^{\dagger}$ & $\mathrm{BCF} \mathrm{Pb}$ & $\mathrm{TF} P b$ \\
\hline 0 & 0 & 0 & $0.00 \pm 0.00 p$ & $0.00 \pm 0.001$ & $0.00 \pm 0.000^{\dagger \dagger}$ \\
\hline 0 & 0 & 5 & $0.00 \pm 0.00 p$ & $0.00 \pm 0.001$ & $0.00 \pm 0.000$ \\
\hline 0 & 0 & 10 & $0.00 \pm 0.00 p$ & $0.00 \pm 0.001$ & $0.00 \pm 0.000$ \\
\hline 0 & 0 & 15 & $0.00 \pm 0.00 p$ & $0.00 \pm 0.001$ & $0.00 \pm 0.000$ \\
\hline 0 & 400 & 0 & $0.68 \pm 0.005 \mathrm{ghi}$ & $1.49 \pm 0.02 \mathrm{bcde}$ & $0.45 \pm 0.01 \mathrm{fgh}$ \\
\hline 0 & 400 & 5 & $0.67 \pm 0.03 \mathrm{hij}$ & $1.45 \pm 0.01 \mathrm{hij}$ & $0.46 \pm 0.01$ efg \\
\hline 0 & 400 & 10 & $0.69 \pm 0.01$ efgh & $1.45 \pm 0.008 \mathrm{hij}$ & $0.47 \pm 0.01 \mathrm{cde}$ \\
\hline 0 & 400 & 15 & $0.69 \pm 0.009$ defgh & $1.43 \pm 0.02 \mathrm{j}$ & $0.48 \pm 0.002 b c$ \\
\hline 0 & 600 & 0 & $0.71 \pm 0.01 \mathrm{abcde}$ & $1.51 \pm 0.01 \mathrm{abcd}$ & $0.46 \pm 0.004 \mathrm{defg}$ \\
\hline 0 & 600 & 5 & $0.71 \pm 0.01 \mathrm{abcd}$ & $1.52 \pm 0.03 a b$ & $0.46 \pm 0.005 \mathrm{defg}$ \\
\hline 0 & 600 & 10 & $0.72 \pm 0.02 a$ & $1.53 \pm 0.01 \mathrm{a}$ & $0.47 \pm 0.01$ cdef \\
\hline 0 & 600 & 15 & $0.66 \pm 0.02 \mathrm{ij}$ & $1.44 \pm 0.01 \mathrm{ij}$ & $0.45 \pm 0.01 \mathrm{fgh}$ \\
\hline 15 & 0 & 0 & $0.00 \pm 0.00 p$ & $0.00 \pm 0.001$ & $0.00 \pm 0.000$ \\
\hline 15 & 0 & 5 & $0.00 \pm 0.00 p$ & $0.00 \pm 0.001$ & $0.00 \pm 0.000$ \\
\hline 15 & 0 & 10 & $0.00 \pm 0.00 p$ & $0.00 \pm 0.001$ & $0.00 \pm 0.000$ \\
\hline 15 & 0 & 15 & $0.00 \pm 0.00 p$ & $0.00 \pm 0.001$ & $0.00 \pm 0.000$ \\
\hline 15 & 400 & 0 & $0.65 \pm 0.01 \mathrm{jk}$ & $1.46 \pm 0.02 \mathrm{fghij}$ & $0.45 \pm 0.01 \mathrm{hi}$ \\
\hline 15 & 400 & 5 & $0.68 \pm 0.006 \mathrm{fghi}$ & $1.45 \pm 0.01 \mathrm{ghij}$ & $0.47 \pm 0.02$ def \\
\hline 15 & 400 & 10 & $0.71 \pm 0.01 \mathrm{abc}$ & $1.45 \pm 0.009 h i j$ & $0.49 \pm 0.004 a b$ \\
\hline 15 & 400 & 15 & $0.70 \pm 0.008 \mathrm{cdefg}$ & $1.39 \pm 0.01 \mathrm{k}$ & $0.50 \pm 0.01 \mathrm{a}$ \\
\hline 15 & 600 & 0 & $0.67 \pm 0.009 \mathrm{ij}$ & $1.51 \pm 0.02 \mathrm{abcd}$ & $0.44 \pm 0.0006 \mathrm{ij}$ \\
\hline 15 & 600 & 5 & $0.67 \pm 0.003 i j$ & $1.43 \pm 0.01 \mathrm{ij}$ & $0.46 \pm 0.003$ efg \\
\hline 15 & 600 & 10 & $0.70 \pm 0.003 \mathrm{bcdef}$ & $1.46 \pm 0.004 f g h i j$ & $0.48 \pm 0.002 \mathrm{bcd}$ \\
\hline 15 & 600 & 15 & $0.72 \pm 0.01 \mathrm{ab}$ & $1.52 \pm 0.03 a b c$ & $0.47 \pm .006 \mathrm{cde}$ \\
\hline 30 & 0 & 0 & $0.00 \pm 0.00 p$ & $0.00 \pm 0.001$ & $0.00 \pm 0.000$ \\
\hline 30 & 0 & 5 & $0.00 \pm 0.00 p$ & $0.00 \pm 0.001$ & $0.00 \pm 0.000$ \\
\hline 30 & 0 & 10 & $0.00 \pm 0.00 p$ & $0.00 \pm 0.001$ & $0.00 \pm 0.000$ \\
\hline 30 & 0 & 15 & $0.00 \pm 0.00 p$ & $0.00 \pm 0.001$ & $0.00 \pm 0.000$ \\
\hline 30 & 400 & 0 & $0.57 \pm 0.01 \mid$ & $1.46 \pm 0.03 \mathrm{ghij}$ & $0.39 \pm 0.01 \mathrm{k}$ \\
\hline 30 & 400 & 5 & $0.58 \pm 0.021$ & $1.49 \pm 0.03 \mathrm{bcdef}$ & $0.39 \pm 0.01 \mathrm{k}$ \\
\hline 30 & 400 & 10 & $0.57 \pm 0.011$ & $1.48 \pm 0.01$ defgh & $0.38 \pm 0.01 \mathrm{k}$ \\
\hline 30 & 400 & 15 & $0.64 \pm 0.006 \mathrm{k}$ & $1.49 \pm 0.02 \mathrm{cdefg}$ & $0.43 \pm 0.008 j$ \\
\hline 30 & 600 & 0 & $0.45 \pm 0.0040$ & $1.50 \pm 0.009 \mathrm{abcd}$ & $0.30 \pm 0.004$ \\
\hline 30 & 600 & 5 & $0.47 \pm 0.003 n$ & $1.47 \pm 0.01$ efghi & $0.32 \pm 0.005 \mathrm{~lm}$ \\
\hline 30 & 600 & 10 & $0.46 \pm 0.001 \mathrm{no}$ & $1.49 \pm 0.02 \mathrm{bcde}$ & $0.31 \pm 0.003 \mathrm{mn}$ \\
\hline 30 & 600 & 15 & $0.50 \pm 0.004 \mathrm{~m}$ & $1.53 \pm 0.02 \mathrm{a}$ & $0.32 \pm 0.006 \mathrm{l}$ \\
\hline
\end{tabular}

† BAC: biological accumulation coefficient; BCF: biological concentration factor; TF: translocation factor.

tt Values followed in each column by the same letter were not significantly different at $5 \%$ level. 
Heavy metal contaminations also affected the biological quality of rhizosphere. So, the increase of the vermicompost, lead, and cadmium enhanced the impacts on microbial respiration in soil. Maximum microbial respiration was observed in the soil containing $30 \mathrm{~kg} \mathrm{~m}^{-2}$ vermicompost, $600 \mathrm{ppm}$ lead,

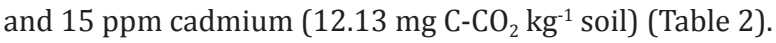

Phytoremediation indices were also influenced by the vermicompost content of the soil (Tables 3 and 4). By increase of vermicompost, the BAC for cadmium and lead was decreased, but increasing this organic matter just enhanced the BCF for cadmium. The metals concentration was also effective on these two factors. BAC factor for both lead and cadmium were increased upon enhancement of the metal content of the soil. In the case of BCF, different results were obtained. Increasing the lead and cadmium concentration in soil caused an increase and a decrease in BCF, respectively. Elevation of cadmium concentration from $0 \mathrm{ppm}$ to $15 \mathrm{ppm}$, just caused a reduction in BCF of the lead. In other words,

TABLE 4. Effect of vermicompost amendment on BAC, BCF, TF and Ti parameters for cadmium in ornamental sunflower under lead $(\mathrm{Pb})$ and cadmium $(\mathrm{Cd})$ stresses. Values are mean $\pm \mathrm{SD}$ of three replicates. In each column, numbers followed by the same letter are not significantly different according to Duncan's test $(\mathrm{P}<0.05)$. $^{\dagger}$

\begin{tabular}{|c|c|c|c|c|c|c|}
\hline $\begin{array}{l}\text { Vermicompost } \\
\left(\mathrm{kg} \mathrm{m}^{-2}\right)\end{array}$ & $\begin{array}{l}\mathrm{Pb} \\
(\mathrm{ppm})\end{array}$ & $\begin{array}{c}\mathrm{Cd} \\
(\mathrm{ppm})\end{array}$ & $\mathrm{BAC} \mathrm{Cd}$ & BCF Cd & TF Cd & $\mathrm{Ti}$ \\
\hline 0 & 0 & 0 & $0.00 \pm 0.00 \mathrm{k}$ & $0.00 \pm 0.00 n$ & $0.00 \pm 0.00 \mathrm{~m}$ & $1.00 \pm 0.00 \mathrm{efgh}^{\dagger \dagger}$ \\
\hline 0 & 0 & 5 & $0.89 \pm 0.13 b c$ & $2.48 \pm 0.2 \mathrm{cde}$ & $0.35 \pm 0.02 \mathrm{ghi}$ & $0.86 \pm 0.45$ efgh \\
\hline 0 & 0 & 10 & $0.57 \pm 0.01 \mathrm{fg}$ & $1.57 \pm 0.03 \mathrm{jk}$ & $0.36 \pm 0.01 \mathrm{ghi}$ & $0.45 \pm 0.20 \mathrm{gh}$ \\
\hline 0 & 0 & 15 & $0.80 \pm 0.05 \mathrm{de}$ & $2.05 \pm 0.04 \mathrm{i}$ & $0.39 \pm 0.01 \mathrm{def}$ & $1.03 \pm 0.87$ efgh \\
\hline 0 & 400 & 0 & $0.00 \pm 0.00 \mathrm{k}$ & $0.00 \pm 0.00 n$ & $0.00 \pm 0.00 \mathrm{~m}$ & $0.18 \pm 0.14 h$ \\
\hline 0 & 400 & 5 & $0.89 \pm 0.07 b c$ & $2.24 \pm 0.10 f g h$ & $0.39 \pm 0.01 \mathrm{def}$ & $0.11 \pm 0.005 \mathrm{~h}$ \\
\hline 0 & 400 & 10 & $0.55 \pm 0.02 f g h$ & $1.50 \pm 0.01 \mathrm{kl}$ & $0.36 \pm 0.01 \mathrm{fgh}$ & $0.16 \pm 0.03 h$ \\
\hline 0 & 400 & 15 & $0.85 \pm 0.02 \mathrm{~cd}$ & $2.05 \pm 0.03 i$ & $0.41 \pm 0.01 \mathrm{~cd}$ & $0.60 \pm 0.03 \mathrm{fgh}$ \\
\hline 0 & 600 & 0 & $0.00 \pm 0.00 \mathrm{k}$ & $0.00 \pm 0.00 n$ & $0.00 \pm 0.00 \mathrm{~m}$ & $0.77 \pm 0.80 \mathrm{fgh}$ \\
\hline 0 & 600 & 5 & $0.98 \pm .0 .01 a$ & $2.05 \pm 0.06 i$ & $0.48 \pm 0.02 a$ & $0.38 \pm 0.15 \mathrm{gh}$ \\
\hline 0 & 600 & 10 & $0.56 \pm 0.02 \mathrm{fgh}$ & $1.57 \pm 0.03 \mathrm{jk}$ & $0.36 \pm 0.01 \mathrm{ghi}$ & $0.44 \pm 0.31 \mathrm{gh}$ \\
\hline 0 & 600 & 15 & $0.94 \pm 0.02 \mathrm{ab}$ & $2.03 \pm 0.03 i$ & $0.46 \pm 0.004 a b$ & $0.32 \pm 0.17 \mathrm{gh}$ \\
\hline 15 & 0 & 0 & $0.00 \pm 0.00 \mathrm{k}$ & $0.00 \pm 0.00 n$ & $0.00 \pm 0.00 \mathrm{~m}$ & $0.57 \pm 0.27 f g h$ \\
\hline 15 & 0 & 5 & $0.75 \pm 0.10 \mathrm{e}$ & $2.93 \pm 0.00 \mathrm{a}$ & $0.25 \pm 0.03 \mathrm{k}$ & $6.16 \pm 0.88 a$ \\
\hline 15 & 0 & 10 & $0.50 \pm 0.02 \mathrm{hij}$ & $1.56 \pm 0.00 \mathrm{jk}$ & $0.32 \pm 0.01 \mathrm{j}$ & $6.20 \pm 1.78 a$ \\
\hline 15 & 0 & 15 & $0.95 \pm 0.01 a b$ & $2.27 \pm 0.01 \mathrm{fg}$ & $0.41 \pm 0.008 \mathrm{~cd}$ & $3.60 \pm 2.70 \mathrm{bcde}$ \\
\hline 15 & 400 & 0 & $0.00 \pm 0.00 \mathrm{k}$ & $0.00 \pm 0.00 n$ & $0.00 \pm 0.00 \mathrm{~m}$ & $1.72 \pm 0.85$ defgh \\
\hline 15 & 400 & 5 & $0.90 \pm 0.05 b c$ & $2.41 \pm 0.19 \mathrm{ed}$ & $0.37 \pm 0.05 \mathrm{efg}$ & $1.42 \pm 1.00$ defgh \\
\hline 15 & 400 & 10 & $0.51 \pm 0.009 \mathrm{hij}$ & $1.53 \pm 0.01 \mathrm{jk} \mid$ & $0.33 \pm 0.01 \mathrm{ij}$ & $6.19 \pm 4.94 a$ \\
\hline 15 & 400 & 15 & $0.85 \pm 0.03 \mathrm{~cd}$ & $2.13 \pm 0.03 \mathrm{hi}$ & $0.40 \pm 0.009 \mathrm{de}$ & $1.75 \pm 0.94$ defgh \\
\hline 15 & 600 & 0 & $0.00 \pm 0.00 \mathrm{k}$ & $0.00 \pm 0.00 n$ & $0.00 \pm 0.00 \mathrm{~m}$ & $4.66 \pm 1.91 \mathrm{abc}$ \\
\hline 15 & 600 & 5 & $0.93 \pm 0.04 a b$ & $2.27 \pm 0.10 \mathrm{fg}$ & $0.41 \pm 0.003 d$ & $6.41 \pm 1.65 a$ \\
\hline 15 & 600 & 10 & $0.59 \pm 0.003 f$ & $1.65 \pm 0.05 j$ & $0.36 \pm 0.01 \mathrm{ghi}$ & $2.28 \pm 0.57 \mathrm{cdefgh}$ \\
\hline 15 & 600 & 15 & $0.90 \pm 0.01 b c$ & $2.03 \pm 0.04 i$ & $0.44 \pm 0.005 b c$ & $0.30 \pm 0.14 \mathrm{gh}$ \\
\hline 30 & 0 & 0 & $0.00 \pm 0.00 \mathrm{k}$ & $0.00 \pm 0.00 n$ & $0.00 \pm 0.00 \mathrm{~m}$ & $2.53 \pm 1.037 \mathrm{cdefgh}$ \\
\hline 30 & 0 & 5 & $0.51 \pm 0.04 \mathrm{hij}$ & $2.64 \pm 0.22 b$ & $0.19 \pm 0.003 \mid$ & $5.76 \pm 0.44 a b$ \\
\hline 30 & 0 & 10 & $0.44 \pm 0.01 \mathrm{j}$ & $1.24 \pm 0.04 m$ & $0.35 \pm 0.02 \mathrm{ghi}$ & $1.55 \pm 1.22$ defgh \\
\hline 30 & 0 & 15 & $0.94 \pm 0.04 a b$ & $2.52 \pm 0.05 \mathrm{bcd}$ & $0.37 \pm 0.01$ efg & $2.51 \pm 1.90 \mathrm{cdefgh}$ \\
\hline 30 & 400 & 0 & $0.00 \pm 0.00 \mathrm{k}$ & $0.00 \pm 0.00 n$ & $0.00 \pm 0.00 \mathrm{~m}$ & $4.08 \pm 2.22 \mathrm{abcd}$ \\
\hline 30 & 400 & 5 & $0.80 \pm 0.04 \mathrm{de}$ & $2.58 \pm 0.02 b c$ & $0.31 \pm 0.02 j$ & $3.22 \pm 2.34 \mathrm{bcdef}$ \\
\hline 30 & 400 & 10 & $0.45 \pm 0.02 \mathrm{ij}$ & $1.41 \pm 0.02$ & $0.32 \pm 0.02 j$ & $3.02 \pm 1.26 \mathrm{cdefg}$ \\
\hline 30 & 400 & 15 & $0.80 \pm 0.01 \mathrm{de}$ & $2.35 \pm 0.06 \mathrm{ef}$ & $0.33 \pm 0.003 \mathrm{hij}$ & $2.89 \pm 0.53 \mathrm{cdefgh}$ \\
\hline 30 & 600 & 0 & $0.00 \pm 0.00 \mathrm{k}$ & $0.00 \pm 0.00 n$ & $0.00 \pm 0.00 \mathrm{~m}$ & $2.46 \pm 1.22 \mathrm{cdefgh}$ \\
\hline 30 & 600 & 5 & $0.98 \pm 0.02 a$ & $2.14 \pm 0.10 \mathrm{ghi}$ & $0.45 \pm 0.02 a b$ & $1.68 \pm 0.64$ defgh \\
\hline 30 & 600 & 10 & $0.53 \pm 0.008 \mathrm{fgh}$ & $1.42 \pm 0.01 \mid$ & $0.37 \pm 0.004 \mathrm{efg}$ & $0.96 \pm 0.32$ efgh \\
\hline 30 & 600 & 15 & $0.84 \pm 0.01 \mathrm{~cd}$ & $2.23 \pm 0.02 \mathrm{fgh}$ & $0.37 \pm 0.007$ efg & $3.34 \pm 0.57$ \\
\hline
\end{tabular}

† BAC: biological accumulation coefficient; BCF: biological concentration factor; TF: translocation factor; Ti: tolerance index.

t+ Values followed in each column by the same letter were not significantly different at $5 \%$ level. 
BCF and BAC for lead were influenced by different concentrations of cadmium rather than lead. Maximum BCF and BAC for lead (0.72) were recorded at the presence of high concentrations of lead (600 ppm) and $10 \mathrm{ppm}$ cadmium without vermicompost amendment. Also, the highest of BAC for cadmium (0.98) was found in the treatment containing $600 \mathrm{ppm}$ lead and $5 \mathrm{ppm}$ cadmium with no vermicompost amendment. The presence of 5-15 ppm cadmium along with low concentrations of lead (0-400 ppm) enhanced the BCF for ornamental sunflower (Tables 2 and 3). As the amount of vermicompost increased, the TF was reduced; but the elevation of metals content in different treatments resulted in a rising trend in this phytoremediation index for both lead and cadmium. The soil amended with $15 \mathrm{~kg} \mathrm{~m}^{-2}$ vermicompost led to the maximum value of TF for lead $(0.5)$ in the treatment containing $400 \mathrm{ppm}$ lead and $15 \mathrm{ppm}$ cadmium. The highest value of TF for cadmium (0.48) was also measured in the treatment involving no vermicompost addition to the soil contaminated by $600 \mathrm{ppm}$ lead and $5 \mathrm{ppm}$ cadmium (Tables 3 and 4). The increase of vermicompost augmented the heavy metal tolerance index, but the elevation of heavy metal concentration caused a decreasing trend of TI. Treating soil with different concentrations of cadmium (up to $10 \mathrm{ppm}$ ) at constant vermicompost and lead contents increased the TI followed by a decline. The highest tolerance index (6.41) was recorded in the treatment involving $15 \mathrm{~kg} \mathrm{~m}^{-2}$ vermicompost, 600 ppm lead and 5 ppm cadmium (Table 4).

\section{Discussion}

The application of vermicompost significantly increased the biomass of Helianthus annuus 'Sungold'. Ornamental sunflower was chosen in this experiment based on their high biomass, fast growth rate and its ability to eliminate heavy metals from contaminated soils. The presence of organic matter in the soil has beneficial influences on the soil and improves its physical and biochemical properties. Thus, vermicompost can increase plant growth and biomass yields (Fu et al., 2011). Sunflower growth and yield were also affected by the stress of the heavy metal (e.g., $\mathrm{Pb}$ and $\mathrm{Cd}$ ). High concentrations of $\mathrm{Pb}$ and $\mathrm{Cd}$ inhibited the growth, reduced biomass production and caused characteristic visible effects similar to those reported by other workers in different plant species (Alaboudi et al., 2018). The decrease in dry matter production as a result of metal stress is in agreement with the earlier studies on plants other than sunflowers (Pandey and Pathak, 2006; Ryser and Sauder, 2006). Decline of sunflower dry and fresh matter as a result of increased $\mathrm{Pb}$ and $\mathrm{Cd}$ content of the soil can be due to the adverse effects of the heavy metals on the ornamental sunflower roots and its translocation into the shoot system, which may reduce the competence of dry matter synthesis and leaf photosynthesis and decrease the dry matter accumulation of the plant. These results were also reported in Helianthus annuus by Zadeh et al. (2008) and Chhotu et al. (2008).

High concentrations of $\mathrm{Pb}$ and $\mathrm{Cd}$ reduced the absorption of the nutrients, preventing the enzymatic activity and inducing oxidative stress, which will alter the enzymes involved in the antioxidant resistance system (Majer et al., 2002; Agrawal and Sharma, 2006). Comparison of the traits showed a significant increase in the CAT and SOD activities in the Helianthus annuus 'Sungold', with an increase of the heavy metals concentration. Such an increase in both enzymes was significantly different from the controls in the treatment involving $600 \mathrm{ppm} \mathrm{Pb}$ and $15 \mathrm{ppm} \mathrm{Cd}$. The maximum responses of these enzymes $(2.18 \%$ and $2.41 \%$ for SOD and CAT, respec- tively) were observed at the maximum concentrations of lead $(600 \mathrm{ppm})$ and cadmium (15 ppm). In the absence of vermicompost, a 27 -fold and 12 -fold increase was observed in SOD and CAT enzymes, respectively at the presence of lead and cadmium ( $600 \mathrm{ppm}$ of $\mathrm{Pb}$ and $15 \mathrm{ppm}$ of $\mathrm{Cd}$ ) when compared to the control. For the same metal concentration, the use of $30 \mathrm{~kg} \mathrm{~m}^{-2}$ vermicompost resulted in 15- and 9-fold increase in SOD and CAT activities, compared to the controls. Oxidant enzymes play a key role in the defense system of the plants against the heavy meta-induced oxidative stresses (Weckx and Clijsters, 1996). Enzymes such as CAT and SOD can deactivate the reactive oxygen species produced in the cells (Mishra et al., 2006; Nair et al., 2015). The increase in the CAT activity could be explained by the adaptive mechanism of the plants to maintain hydrogen peroxide $\left(\mathrm{H}_{2} \mathrm{O}_{2}\right)$ of the cells at a steady level (Mishra et al., 2006). Oxidative stresses induce the production of oxygen free radicals, such as superoxide radicals $\left(\mathrm{O}^{-}\right), \mathrm{H}_{2} \mathrm{O}_{2}$ and hydroxyl radicals $(\mathrm{OH})$ and free radicals hydroxyl respond resulting in the fat peroxidation (Chen et al., 2007). In the present study, CAT and SOD activity was significantly increased in leaves of $\mathrm{Pb}$ - and $\mathrm{Cd}$-treated plants compared to the controls, which perhaps indicates the breakdown of $\mathrm{H}_{2} \mathrm{O}_{2}$ and toxic peroxides by heavy metal accumulation. Increase of CAT and SOD activity in response to $\mathrm{Cd}$ and $\mathrm{Pb}$ was reported in Vigna radiate L. Wilczek (Anjum et al. 2008), Jatropha curcas L., (Shu et al., 2012), Pisum sativum L. (Rodríguez-Serrano et al., 2006), and Morus alba (Tewari et al., 2013) as well. In contrast, addition of vermicompost significantly reduced the activity of CAT and SOD enzymes. The protective effect of vermicompost application might be due to its ability to prevent $\mathrm{Pb}$ and $\mathrm{Cd}$ transfer to the aerial organs, adjust the heavy metals stress effect, and inhibit oxygen-centered free radical generation, thereby preventing the initiation of ROS formation (Aravind and Prasad, 2005). It has been also reported that modulation of the antioxidant response may be the main task of fungal symbiont in metal tolerance contribution in the mycorrhizal symbioses as a biotic component of vermicompost (Garg and Kaur, 2013).

Apparently, vermicompost application significantly affected microbial respiration, especially in the Pb-contaminated. The addition of organic amendments has been shown to increase the soil microbial activity (Chander and Joergensen, 2002). As the microbial role of soil is essential in the nutrient turnover and plant nutrition, further research is required to assess the changes in the subsurface microbial activities. Our results generally agree with Farrell et al. (2009), who found that compost amendment can significantly affect the remediation strategy in metal-contaminated soils.

The BAC, BCF, and TF have been usually used to evaluate the translocations of heavy metals into the plant's tissues. The BCF and TF reflect the plant's potential to tolerate and accumulate heavy metals in their organs as well as their ability to phytoextraction (Barbafieri et al., 2017). TF values greater than 1 indicate the heavy metal translocation from root to the aerial organs (Galal and Shehata, 2015). The results showed that the TF was not greater than 1 in ornamental sunflower at any of the studied treatments, but the BCF exceeded 1 in the examined samples, with the exception of the pots free from $\mathrm{Pb}$ and $\mathrm{Cd}$. Therefore, this species is unsuitable for $\mathrm{Pb}$ and Cd phytoextraction due to $\mathrm{TF}<1$ and $\mathrm{BCF}>1$ (Sidhu et al., 2017), but ornamental sunflower can be a proper candidate for phytostabilization of $\mathrm{Pb}$ and $\mathrm{Cd}$ at the examined concentrations. The TF factor decreased with the application of 15 to $30 \mathrm{~kg} \mathrm{~m}^{-2}$ vermicompost compared to others. This founding indicated that $\mathrm{Pb}$ and $\mathrm{Cd}$ concentrations in the shoot of 
ornamental sunflower decreased with the addition of vermicompost due to a decline in the extractable Cd content of the soil. This could be attributed to the heavy metals bound by organic ligands in vermicompost (Wei et al., 2011; Wang et al., 2013). The organic amendment led to an effective immobilization of some heavy metals such as $\mathrm{Pb}, \mathrm{Cu}, \mathrm{Zn}$ and $\mathrm{Cd}$ in soil (Angelova et al., 2016). Furthermore, the extended roots of ornamental sunflower and intensive dispersion of roots into the soil decreased the leaching via stabilization; hence immobilizing and concentrating $\mathrm{Pb}$ and $\mathrm{Cd}$ in the roots. The results agreed with those obtained by Angelova et al. (2016) who found that the organic amendments led to decreased $\mathrm{Pb}$ and $\mathrm{Cu}$ content in the potato peel and tubers. They also observed that at higher $\mathrm{Cd}$ concentrations, the amount of added amendment was not sufficient to immobilize all the available $\mathrm{Cd}$. The presence of organic carbon increases the cation exchangeability of the soil which retains the nutrients assimilated by plants (Yobouet et al., 2010). Increasing the amount of organic matter in the soil helps to minimize the absorption of heavy metals by the plants (Fijalkowski et al., 2012). Organic amendments to contaminated soils (containing labile elements) reduced the overall bio-availabilities of the metals due to sorption processes (Brown et al., 2003).

The obtained results showed that ornamental sunflower can accumulate $\mathrm{Pb}$ and $\mathrm{Cd}$ in its tissues. However, the accumulation of $\mathrm{Pb}$ in the plant shoot was more favorable than $\mathrm{Cd}$. Considering the potential for $\mathrm{Pb}$ phytoextraction, sunflower exhibits promising characteristics, as previously reported by other authors (Seth et al., 2011; Kacálková et al., 2014; Sewalem et al., 2014; Alaboudi et al., 2018; and Pilon-Smits, 2005). Computed TI was higher than 1 in the ornamental sunflower grown in the vermicompost-containing $\mathrm{Pb}$ - and $\mathrm{Cd}$-contaminated soil. TI values were above one or approximately one; confirming the non-toxicity of heavy metals on the plant biomass. Gondek (2008) found that composts and vermicompost increased the tolerance characteristics of the plants cultivated in Cd-contaminated soils, which is consistent with the results of this study.

H. annuus 'Sungold' plant showed tolerance toward $\mathrm{Pb}$ and Cd contamination and this ornamental plant was more favorable for $\mathrm{Pb}$ uptake compared to $\mathrm{Cd}$. Therefore, it can be recommended for the remediation of $\mathrm{Pb}$ - and $\mathrm{Cd}$-contaminated soils.

\section{References}

Aebi, H. (1984). Catalase in vitro. Meth. Enzymol. 105, 121-126. https://doi.org/10.1016/S0076-6879(84)05016-3.

Agrawal, V., and Sharma, K. (2006). Phytotoxic effects of $\mathrm{Cu}, \mathrm{Zn}, \mathrm{Cd}$ and $\mathrm{Pb}$ on in vitro regeneration and concomitant protein changes in Holarrhena antidysenteric. J. Biotechnol. Plant. 50, 307-310. https:// doi.org/10.1007/s10535-006-0027-z.

Alaboudi, K.A., Ahmed, B., and Brodie, G. (2018). Phytoremediation of $\mathrm{Pb}$ and $\mathrm{Cd}$ contaminated soils by using sunflower (Helianthus annuus) plant. Ann. Agric. Sci. 63(1), 123-127. https://doi. org/10.1016/j.aoas.2018.05.007.

Alef, K. (1995). Soil respiration. In Methods in Applied Soil Microbiology and Biochemistry, K. Alef, and P. Nannipieri, eds. (London, San Diego, New York, Boston, Sydney, Tokyo, and Toronto: Harcourt Brace \& Company Publishers), p. 214-216.

Angelova, V.R., Ivanova, R.V., Ivanov, K.I., Perifanova-Nemska, M.N., and Uzunova, G.I. (2016). Potential of sunflower (Helianthus annuus L.) for phytoremediation of soils contaminated with heavy metals. World J. Sci. Engin. Technol. 10, 1-11.
Anjum, N.A., Umar, S., Ahmad, A., and Iqbal, M. (2008). Responses of components of antioxidant system in moongbean genotypes to cadmium stress. Commun. Soil Sci. Plant Anal. 39(15-16), 24692483. https://doi.org/10.1080/00103620802292871.

Aravind, P., and Prasad, M.N.V. (2005). Modulation of cadmiuminduced oxidative stress in Ceratophyllum demersum by zinc involves ascorbate-glutathione cycle and glutathione metabolism. Plant Physiol. Biochem. 43(2), 107-116. https://doi.org/10.1016/j. plaphy.2005.01.002.

Balestrasse, K.B., Gallego, S.M., and Tomaro, M.L. (2006). Oxidation of the enzymes involved in nitrogen assimilation plays an important role in the cadmium-induced toxicity in soybean plants. Plant Soil 284(1-2), 187-194. https://doi.org/10.1007/s11104-006-0050-z.

Barbafieri, M., Pedron, F., Petruzzelli, G., Rosellini, I., Franchi, E., Bagatin, R., and Vocciante, M. (2017). Assisted phytoremediation of a multi-contaminated soil: investigation on arsenic and lead combined mobilization and removal. J. Environm. Manag. 203, 316-329. https://doi.org/10.1016/j.jenvman.2017.07.078.

Brown, S.L., Henry, C.L., Chaney, R., Compton, H., and De Volder, P.S. (2003). Using municipal biosolids in combination with other residuals to restore metal-contaminated mining areas. Plant Soil 249, 203-215.

Chander, K., and Joergensen, R.G. (2002). Decomposition of ${ }^{14} \mathrm{C}$ labelled glucose in a $\mathrm{Pb}$-contaminated soil remediated with synthetic zeolite and other amendments. Soil Biol. Biochem. 34(5), 643-649. https://doi.org/10.1016/S0038-0717(01)00226-7.

Chen, J., Zhu, C., Lin, D., and Sun, Z.X. (2007). The effects of Cd on lipid peroxidation, hydrogen peroxide content and antioxidant enzyme activities in Cd-sensitive mutant rice seedlings. Canadian J. Plant Sci. 87(1), 49-57. https://doi.org/10.4141/P06-048.

Chhotu, D., Jadia, M., and Fulekar, H. (2008). Phytoremediation: The application of vermicompost to remove zinc, cadmium, copper, nickel and lead by sunflower plant. Environm. Engin. Manag. J. 7(5), 547-558. https://doi.org/10.30638/eemj.2008.078.

Chirakkara, R.A., and Reddy, K.R. (2015). Biomass and chemical amendments for enhanced phytoremediation of mixed contaminated soils. Ecol. Engin. 85, 265-274. https://doi.org/10.1016/j.ecoleng. 2015.09.029.

Farouk, S., Mosa, A.A., Taha, A.A., Ibrahim, H.M., and Gahmery, A.E. (2011). Protective effect of humic acid and chitosan on radish (Raphanus sativus, L. var. sativus) plants subjected to cadmium stress. J. Stress Physiol. Biochem. 7(2), 99-116.

Farrell, M., and Jones, D.L. (2009). Critical evaluation of municipal solid waste composting and potential compost markets. Bioresource Technol. 100(19), 4301-4310. https://doi.org/10.1016/j.biortech. 2009.04.029.

Fijalkowski, K., Kacprzak, M., Grobelak, A., and Placek, A. (2012). The influence of selected soil parameters on the mobility of heavy metals in soils. Inzynieria i Ochrona Srodowiska 5, 81-92.

Fu, D.Q., Teng, Y., Luo, Y.M., Tu, C., Li, S.X., Li, Z.G., and Christie, P. (2011). Effects of alfalfa and organic fertilizer on benzo[a]pyrene dissipation in an aged contaminated soil. Environm. Sci. Pollut. Res. 19(5), 1605-1611. https://doi.org/10.1007/s11356-011-0672-4.

Galal, T.M., and Shehata, H.S. (2015). Bioaccumulation and translocation of heavy metals by Plantago major L. grown in contaminated soils under the effect of traffic pollution. Ecological Indicators 48, 244-251. https://doi.org/10.1016/j.ecolind.2014.08.013.

Garg, N., and Kaur, H. (2013). Response of antioxidant enzymes, phytochelatins and glutathione production towards $\mathrm{Cd}$ and $\mathrm{Zn}$ stresses in Cajanus cajan (L.) Millsp. genotypes colonized by arbuscular mycorrhizal fungi. J. Agron. Crop Sci. 199(2), 118-133. https://doi.org/10.1111/j.1439-037X.2012.00533.x. 
Ghori, Z., Iftikar, H, Bhatti, M.F., Minullah, N., Sharma, I., Kazi, A.G., and Ahmad, P. (2016). Phytoextraction: The use of plants to remove heavy metals from soil. Plant Metal Interaction 385-409. https:// doi.org/10.1016/B978-0-12-803158-2.00015-1.

Giri, A.K., and Patel, R.K. (2012). Phytoaccumulation potential and toxicity of arsenic ions by Eichhornia crassipes in hydroponic system. J. Bioremed. Biodegrad. 3(2), 137. https://doi.org/10.4172/21556199.1000137.

Gondek, K. (2008). Chromium bioaccumulation from composts and vermicomposts based on tannery. J. Central Europ. Agric. 9(1), 129-140.

Govarthanana, M., Mythilib, R., Selvankumarb, T., Kamala-Kannanc, S., and Kima, H. (2018). Myco-phytoremediation of arsenic- and lead-contaminated soils by Helianthus annuus and wood rot fungi, Trichoderma sp. isolated from decayed wood. Ecotoxicol. Environm. Safety 151, 279-284. https://doi.org/10.1016/j. ecoenv.2018.01.020.

Kacálková, L., Tlustoš, P., and Száková, J. (2009). Phytoextraction of cadmium, copper, zinc and mercury by selected plants. Plant, Soil, Environm. 7, 295-304. https://doi.org/10.17221/100/2009-PSE.

Lasat, M.M. (2002). Phytoextraction of toxic metals. J. Environm. Quality 31(1), 109-120. https://doi.org/10.2134/jeq2002.1090.

Lindsay, W.L., and Norvell, W.A. (1978). Development of a DTPA soil test for zinc, iron, manganese, and copper. Soil Sci. Soc. Am. J. 42(3), 421-428. https://doi.org/10.2136/ sssaj1978.03615995004200030009x.

Majer, B.J., Tscherko, D., and Paschke, A. (2002). Effects of heavy metal contamination of soils on micronucleus induction in Tradescantia and on microbial enzyme activities: A comparative investigation. Mutat. Res. 515, 111-124. https://doi.org/10.1016/ S1383-5718(02)00004-9.

Martínez-Fernández, D., and Walker, D.J. (2012). The effects of soil amendments on the growth of Atriplex halimus and Bituminaria bituminosa in heavy metal-contaminated soils. Water, Air, Soil Pollution 223(1), 63-72. https://doi.org/10.1007/s11270-011-0839-0.

Mishra, S., Srivastava, S., Tripathi, R.D., Kumar, P., Seth, C.S., and Gupta, D.K. (2006). Lead detoxification by coontail (Ceratophyllum demersum L.) involves induction of phytochelatins and antioxidant system in response to its accumulation. Chemosphere 65, 10271039. https://doi.org/10.1016/j.chemosphere.2006.03.033.

Muszynska, E., and Hanus-Fajerska, E. (2015). Why are heavy metal hyperaccumulating plants so amazing? BioTechnologia, J. Biotechnol. Comput. Biol. Bionanotechnol. 96(4). https://doi.org/10.5114/ bta.2015.57730.

Nair, A.R., Lee, W.K., Smeets, K., Swennen, Q., Sanchez, A., Thévenod, F., and Cuypers, A. (2015). Glutathione and mitochondria determine acute defense responses and adaptive processes in cadmiuminduced oxidative stress and toxicity of the kidney. Arch. Toxicol. 89(12), 2273-2289. https://doi.org/10.1007/s00204-014-1401-9.

Pandey, N., and Pathak, G.C. (2006). Nickel alters antioxidative defence and water status in greengram. Indian J. Plant Physiol. 11, 113-118.

Pilon-Smits, E. (2005). Phytoremediation. Annual Rev. Plant Biol. 56, 15-39. https://doi.org/10.1146/annurev.arplant.56.032604.144214.

Rios-Gonzales, K., Erdei, L., and Lips, S.H. (2002). The activity of antioxidant enzymes in maize and sunflower seedlings as affected by salinity and different nitrogen sources. Plant Sci. 162, 923-930. https://doi.org/10.1016/S0168-9452(02)00040-7.

Rizwan, M., Ali, S., Rizvi, H., Rinklebe, J., Tsang, D.C.W., Meers, E., Ok, Y.S., and Ishaque, W. (2016). Phytomanagement of heavy metals in contaminated soils using sunflower - A review. Crit. Rev. Environm. Sci. Technol. 46, 1498-1528. https://doi.org/10.1080/10643389.2 016.1248199 .
Rodríguez-Serrano, M., Romero-Puertas, M.C., Zabalza, A., Corpas, F.J., Gómez, M., del Río, L.A., and Sandalio, L.M. (2006). Cadmium effect on oxidative metabolism of pea (Pisum sativum L.) roots. Imaging of reactive oxygen species and nitric oxide accumulation in vivo. Plant, Cell, Environm. 29(8), 1532-1544. https://doi.org/10.1111/j.13653040.2006.01531.x.

Ryser, P., and Sauder, W.R. (2006). Effects of heavy-metalcontaminated soil on growth, phenology and biomass turnover of Hieracium piloselloides. Environm. Pollut. 140, 52-61. https://doi. org/10.1016/j.envpol.2005.06.026.

Sabienë, N., Brazauskienë, D.M., and Rimmer, D. (2004). Determination of heavy metal mobile forms by different extraction methods. Ekologija 1, 36-41.

Sandalio, L.M., Serrano, M.R., del Rio, L.A., and Romero-Puertas, M.C. (2009). Reactive oxygen species and signaling in cadmium toxicity. In Reactive Oxygen Species in Plant Signaling. Signaling and Communication in Plants, L.A. del Rio, and A. Puppo, eds. (Berlin, Heidelberg, Germany: Springer Verlag), p. 175-189. https://doi. org/10.1007/978-3-642-00390-5_11.

Seth, C.S., Misra, V., Singh, R.R., and Zolla, L. (2011). EDTAenhanced lead phytoremediation in sunflower (Helianthus annuus L.) hydroponic culture. Plant Soil 347, 231-242. https://doi. org/10.1007/s11104-011-0841-8.

Sewalem, N., Elfeky, S., and Shintinawy, F.E. (2014). Phytoremediation of lead and cadmium contaminated soils using sunflower plant phytoremediation of lead and cadmium contaminated soils using sunflower plant. J. Stress Physiol. Biochem. 10, 122-134.

Shu, X., Yin, L., Zhang, Q., and Wang, W. (2012). Effect of Pb toxicity on leaf growth, antioxidant enzyme activities, and photosynthesis in cuttings and seedlings of Jatropha curcas L. Environm. Sci. Pollut. Res. 19(3), 893-902. https://doi.org/10.1007/s11356-011-0625-y.

Sidhu, G.P.S., Singh, H.P., Batish, D.R., and Kohli, R.K. (2017). Tolerance and hyperaccumulation of cadmium by a wild, unpalatable herb Coronopus didymus (L.) Sm. (Brassicaceae). Ecotoxicol. Environm. Safety 135, 209-215.https://doi.org/10.1016/j.ecoenv.2016.10.001.

Singer, A.C., Bell, T., Heywood, C.A., Smith, J.A.C., and Thompson, I.P. (2007). Phytoremediation of mixed-contaminated soil using the hyperaccumulator plant Alyssum lesbiacum: Evidence of histidine as a measure of phytoextractable nickel. Environm. Pollut. 147, 74-82. https://doi.org/10.1016/j.envpol.2006.08.029.

Taghizadeh, M., Kafi, M., Fatahi, F., and Savaghebi, G. (2012). Effects of lead concentrations on seed germination of turfgrass genus and its potential for phytoremediation. Iranian J. Hortic. Sci. 42(3), 276-289.

Tewari, R.K., Kumar, P., and Sharma, P.N. (2013). Oxidative stress and antioxidant responses of mulberry (Morus alba) plants subjected to deficiency and excess of manganese. Acta Physiol. Plant. 35(12), 3345-3356. https://doi.org/10.1007/s11738-013-1367-x.

Ullah, A., Mushtaq, H., Ali, H., Munis, M., Javed, M.T., and Chudhary, H.J. (2015). Diazotrophs-assisted phytoremediation of heavy metals: a novel approach. Environm. Sci. Pollut. Res. 22, 2505-2514. https:// doi.org/10.1007/s11356-014-3699-5.

Wang, K., Huang, H., Zhu, Z., Li, T., He, Z., Yang, X., and Alva, A. (2013). Phytoextraction of metals and rhizoremediation of PAHs in cocontaminated soil by co-planting of Sedum alfredii with ryegrass (Lolium perenne) or castor (Ricinus communis). Int. J. Phytoremed. 15(3), 283-298. https://doi.org/10.1080/15226514.2012.694501.

Weckx, J.E., and Clijsters, H.M. (1996). Oxidative damage and defense mechanisms in primary leaves of Phaseolus vulgaris as a result of root assimilation of toxic amounts of copper. Physiol. Plant. 96, 506512. https://doi.org/10.1034/j.1399-3054.1996.960322.x. 
Wei, S., Zhu, J., Zhou, Q.X., and Zhan, J. (2011). Fertilizer amendment for improving the phytoextraction of cadmium by a hyperaccumulator Rorippa globosa (Turcz.) Thell. J. Soils Sediments 11(6), 915. https:// doi.org/10.1007/s11368-011-0389-5.

Yobouet, Y.A., Adouby, K., Trokourey, A., and Yao, B. (2010). Cadmium, copper, lead and zinc speciation in contaminated soils. Int. J. Engin. Sci. Technol. 2, 802-812.

Zadeh, B.M., Savaghebi-Firozabadi, G.R., Alikhani, H.A., and Hosseini, H.M. (2008). Effect of sunflower and amaranthus culture and application of inoculants on phytoremediation of the soils contaminated with cadmium. Am. Eurasian J. Agric. Environm. Sci. 4(1), 93-103.

Received: Aug. 26, 2019

Accepted: Dec. 6, 2019

Addresses of authors:

Fatemeh Mojdehi ${ }^{1}$, Mina Taghizadeh ${ }^{2, *}$,

Amir Hossein Baghaie ${ }^{3}$, Mahdi Changizi ${ }^{4}$ and

Shahab Khaghani ${ }^{4}$

${ }^{1}$ Department of Horticultural Science, Arak Branch, Islamic Azad University, Arak, Iran

2 Department of Horticultural Science, Faculty of Agriculture and Environment Science, Arak University, Arak, Iran

${ }^{3}$ Department of Soil Science, Arak Branch, Islamic Azad University, Arak, Iran

${ }^{4}$ Department of Agronomy and Plant Breeding, Arak Branch, Islamic Azad University, Arak, Iran

* Corresponding author;

E-mail: m-taghizadeh@araku.ac.ir

Tel.: +989124666143 